\title{
On Multiplicative Generators of n-Dimensional Overlap Functions
}

\author{
Hai Xie ${ }^{1,2}$ \\ ${ }^{1}$ College of Science, Guilin University of Technology, Guilin, China \\ ${ }^{2}$ Center for Data Analysis and Algorithm Technology, Guilin University of Technology, Guilin, China \\ Email: xiehai126@126.com
}

How to cite this paper: Xie, H. (2020) On Multiplicative Generators of n-Dimensional Overlap Functions. Applied Mathematics, 11, 1061-1069.

https://doi.org/10.4236/am.2020.1111071

Received: July 30, 2020

Accepted: November 1, 2020

Published: November 4, 2020

Copyright $\odot 2020$ by author(s) and Scientific Research Publishing Inc. This work is licensed under the Creative Commons Attribution International License (CC BY 4.0).

http://creativecommons.org/licenses/by/4.0/

\begin{abstract}
In this paper, inspired by the multiplicative generators of overlap functions, we mainly propose the concepts of multiplicative generator pairs of n-dimensional overlap functions, in order to extend the dimensionality of overlap functions from 2 to $\mathrm{n}$. We present the condition under which the pair $(\mathbf{g}, \mathbf{h})$ can multiplicatively generate an n-dimensional overlap function $\boldsymbol{O}_{\mathbf{g}, \mathbf{h}}$. we focus on the homogeneity and idempotency property on multiplicatively generated $\mathrm{n}$-dimensional overlap functions.
\end{abstract}

\section{Keywords}

n-Dimensional Overlap Functions, Overlap Functions, Multiplicative Generators

\section{Introduction}

Overlap functions [1] [2] and grouping functions [3] are two particular cases of bivariate continuous aggregation functions. Those two concepts have been applied to some interesting problems, for example, image processing, classification or decision making. In [4], Gómez et al. introduced the definition of n-dimensional overlap functions and the conditions under which n-dimensional overlap functions are migrative, homogeneous or Lipschitz continuous. In [5], Dimuro et al. introduced the notion of additive generator pair for overlap functions and analyzed the influence of the migrativity, homogeneity and idempotency properties in the overlap functions obtained by such distortion and their respective additive generator pairs. Qiao and $\mathrm{Hu}$ [6] proposed the concept of multiplicative generator pair for overlap functions and grouping functions, and investigated the migrativity, homogeneity, idempotency, Archimedean and cancellation properties 
for the overlap functions and grouping functions obtained by such multiplicative generator pairs. The main purpose of [6] is to present one new way to construct overlap function and grouping function by use of multiplicative generator pairs. In a fuzzy classification system, one always need to measure the degree of overlapping of an object with more than two classes. From the theoretical and applied point of view, we need to study how to construct n-dimensional overlap function by use of multiplicative generator pairs. In this paper, we will propose the notions of multiplicative generator pairs of $\mathrm{n}$-dimensional overlap functions. Furthermore, we study the homogeneity and idempotency property on multiplicatively generated $\mathrm{n}$-dimensional overlap functions.

The rest of this paper is organized as follows. In Section 2, we present some basic definitions on overlap functions and $\mathrm{n}$-dimensional overlap functions. We introduce the concept of multiplicative generators of $n$-dimensional overlap functions in Section 3. We study the homogeneity and idempotency property on multiplicatively generated n-dimensional overlap functions in Section 4. Finally, we end this paper with some conclusions.

\section{Preliminaries}

In this section, we recall some basic concepts of overlap functions and $\mathrm{n}$-dimensional overlap functions, which shall be needed in the sequel.

Definition 2.1. (See Bustince et al. [1]) A bivariate function $O:[0,1]^{2} \rightarrow[0,1]$ is said to be an overlap function if it satisfies the following conditions:

$\left(O_{1}\right) O$ is commutative;

$\left(O_{2}\right) O(x, y)=0$ iff $x y=0$;

$\left(O_{3}\right) O(x, y)=1$ iff $x y=1$;

$\left(\mathrm{O}_{4}\right) \mathrm{O}$ is increasing;

$\left(\mathrm{O}_{5}\right) \mathrm{O}$ is continuous.

Definition 2.2. (See Gómez et al. [4]) An n-dimensional aggregation function $\boldsymbol{O}:[0,1]^{n} \rightarrow[0,1]$ is an n-dimensional overlap function if and only if:

$\left(\boldsymbol{O}_{1}\right) \boldsymbol{O}$ is symmetric.

$\left(\boldsymbol{O}_{2}\right) \boldsymbol{O}\left(x_{1}, \cdots, x_{n}\right)=0$ if and only if $\prod_{i=1}^{n} x_{i}=0$.

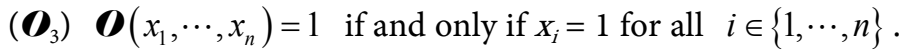

$\left(\boldsymbol{O}_{4}\right) \boldsymbol{O}$ is increasing.

$\left(\boldsymbol{O}_{5}\right) \boldsymbol{O}$ is continuous.

Definition 2.3. (See Dimuro et al. [5]) A function $F:[0,1] \rightarrow[0,1]$ is said to be a pseudo-automorphism if the following conditions hold:

$\left(F_{1}\right) F$ is increasing;

$\left(F_{2}\right) F$ is continuous;

$\left(F_{3}\right) F(x)=1$ iff $x=1$;

$\left(F_{4}\right) F(x)=0$ iff $x=0$.

A function $\varphi:[0,1] \rightarrow[0,1]$ is an automorphism if it is a continuous and strictly increasing function such that $\varphi(0)=0$ and $\varphi(1)=1$ [7]. Obviously, any automorphism is a strictly increasing pseudo-automorphism. 


\section{Multiplicative Generators of n-Dimensional Overlap Functions}

In this section, we try to extend the notion of multiplicative generators of overlap functions to the n-dimensional case, and characterize the basic properties of multiplicative generators of $\mathrm{n}$-dimensional overlap functions.

Definition 3.1. Consider two continuous and increasing functions $\mathbf{g}, \mathbf{h}:[0,1] \rightarrow[0,1]$. The n-dimensional function $\boldsymbol{O}_{\mathbf{g , h}}:[0,1]^{n} \rightarrow[0,1]$ given by

$$
\boldsymbol{O}_{\mathbf{g}, \mathbf{h}}\left(x_{1}, x_{2}, \cdots, x_{n}\right)=\mathbf{g}\left(\mathbf{h}\left(x_{1}\right) \mathbf{h}\left(x_{2}\right) \cdots \mathbf{h}\left(x_{n}\right)\right)
$$

If $\boldsymbol{O}_{\mathbf{g , h}}$ is an n-dimensional overlap function, then $(\mathbf{g}, \mathbf{h})$ is said to be a multiplicative generator pair of the overlap function $\boldsymbol{O}_{\mathbf{g , h}}$ and $\boldsymbol{O}_{\mathbf{g}, \mathbf{h}}$ is called multiplicatively generated by the pair $(\mathbf{g}, \mathbf{h})$.

Proposition 3.1. Consider two continuous and increasing functions

$\mathbf{g}, \mathbf{h}:[0,1] \rightarrow[0,1]$ such that

1) $\mathbf{h}(x)=0$ iff $x=0 ;$
2) $\mathbf{h}(x)=1$ iff $x=1 ;$
3) $\mathbf{g}(x)=0$ iff $x=0 ;$
4) $\mathbf{g}(x)=1$ iff $x=1$.

Then, the n-dimensional function $\boldsymbol{O}_{\mathbf{g}, \mathbf{h}}:[0,1]^{n} \rightarrow[0,1]$, given by

$$
\boldsymbol{O}_{\mathbf{g}, \mathbf{h}}\left(x_{1}, x_{2}, \cdots, x_{n}\right)=\mathbf{g}\left(\mathbf{h}\left(x_{1}\right) \mathbf{h}\left(x_{2}\right) \cdots \mathbf{h}\left(x_{n}\right)\right)
$$

is an n-dimensional overlap function.

Proof. We check out one by one that $\boldsymbol{O}_{\mathbf{g , h}}$ satisfies the conditions of Definition 2.2 as follows.

$\left(\boldsymbol{O}_{1}\right)$ The commutativity is obvious by the definition of $\boldsymbol{O}_{\mathbf{g , h}}$.

$\left(\boldsymbol{O}_{2}\right) \quad \boldsymbol{O}_{\mathbf{g}, \mathbf{h}}\left(x_{1}, x_{2}, \cdots, x_{n}\right)=0 \Leftrightarrow \mathbf{g}\left(\mathbf{h}\left(x_{1}\right) \mathbf{h}\left(x_{2}\right) \cdots \mathbf{h}\left(x_{n}\right)\right)=0$

$\Leftrightarrow \mathbf{h}\left(x_{1}\right) \mathbf{h}\left(x_{2}\right) \cdots \mathbf{h}\left(x_{n}\right)=0$ by item (3)

$\Leftrightarrow \mathbf{h}\left(x_{1}\right)=0$ or $\mathbf{h}\left(x_{2}\right)=0$ or $\cdots$ or $\mathbf{h}\left(x_{n}\right)=0$

$\Leftrightarrow x_{1}=0$ or $x_{2}=0$ or $\cdots$ or $x_{n}=0$ by item (1)

$\Leftrightarrow \quad x_{1} x_{2} \cdots x_{n}=0$.

$\left(\boldsymbol{O}_{3}\right) \quad \boldsymbol{O}_{\mathbf{g}, \mathbf{h}}\left(x_{1}, x_{2}, \cdots, x_{n}\right)=1 \Leftrightarrow \mathbf{g}\left(\mathbf{h}\left(x_{1}\right) \mathbf{h}\left(x_{2}\right) \cdots \mathbf{h}\left(x_{n}\right)\right)=1$

$\Leftrightarrow \mathbf{h}\left(x_{1}\right) \mathbf{h}\left(x_{2}\right) \cdots \mathbf{h}\left(x_{n}\right)=1$ by item (4)

$\Leftrightarrow \mathbf{h}\left(x_{1}\right)=1$ and $\mathbf{h}\left(x_{2}\right)=1$ and $\cdots$ and $\mathbf{h}\left(x_{n}\right)=1$

$\Leftrightarrow \quad x_{1}=1$ and $x_{2}=1$ and $\cdots$ and $x_{n}=1$ byitem (2).

$\left(\boldsymbol{O}_{4}\right)$ By the monotonicity of $\mathbf{g}$ and $\mathbf{h}$, it is easy to get that $\boldsymbol{O}_{\mathbf{g , h}}$ is increasing.

$\left(\boldsymbol{O}_{5}\right)$ From the continuities of $\mathbf{g}$ and $\mathbf{h}$, the continuity can be obtained immediately.

Proposition 3.2. Consider two continuous and increasing functions

$\mathbf{g}, \mathbf{h}:[0,1] \rightarrow[0,1]$ such that

1) $\mathbf{g}(x)=0$ iff $x=0$;

2) $\mathbf{g}(x)=1$ iff $x=1$;

3) $\boldsymbol{O}_{\mathbf{g}, \mathbf{h}}\left(x_{1}, x_{2}, \cdots, x_{n}\right)=\mathbf{g}\left(\mathbf{h}\left(x_{1}\right) \mathbf{h}\left(x_{2}\right) \cdots \mathbf{h}\left(x_{n}\right)\right)$ is an $\mathrm{n}$-dimensional overlap function.

Then the following statements hold: 
1) $\mathbf{h}(\mathrm{x})=0$ iff $\mathrm{x}=0$;

2) $\mathbf{h}(x)=1$ iff $x=1$.

Proof. 1) ( $\Rightarrow)$ If $\mathbf{h}(x)=0$, then $\mathbf{h}(x) \mathbf{h}\left(x_{1}\right) \cdots \mathbf{h}\left(x_{n-1}\right)=0$ for any $x_{i} \in(0,1]$ ( $i=1, \cdots, n-1)$. Furthermore, by items (1) and (3), one can get that

$$
\boldsymbol{O}_{\mathbf{g}, \mathbf{h}}\left(x, x_{1}, \cdots, x_{n-1}\right)=\mathbf{g}\left(\mathbf{h}(x) \mathbf{h}\left(x_{1}\right) \cdots \mathbf{h}\left(x_{n-1}\right)\right)=0 .
$$

Thus, it follows that $x=0$ from $\left(\boldsymbol{O}_{2}\right)$.

$(\Leftarrow)$ If $x=0$, then, by item (3), we can obtain that

$$
\mathbf{g}(\mathbf{h}(x) \mathbf{h}(x) \cdots \mathbf{h}(x))=\boldsymbol{O}_{\mathbf{g}, \mathbf{h}}(x, x, \cdots, x)=0 .
$$

Furthermore, one has that $\mathbf{h}(x)=0$ by item (1). Hence, we get that $\mathbf{h}(x)=0$ iff $x=0$.

2) It can be verified in a similar way as item (1).

Proposition 3.3. Consider two continuous and increasing functions

$\mathbf{g}, \mathbf{h}:[0,1] \rightarrow[0,1]$ and $\mathbf{g}, \mathbf{h}$ such that

1) $\mathbf{h}(x)=0$ iff $x=0$;

2) $\mathbf{h}(x)=1$ iff $x=1$;

3) $\boldsymbol{O}_{\mathbf{g}, \mathbf{h}}\left(x_{1}, x_{2}, \cdots, x_{n}\right)=\mathbf{g}\left(\mathbf{h}\left(x_{1}\right) \mathbf{h}\left(x_{2}\right) \cdots \mathbf{h}\left(x_{n}\right)\right)$ is an $\mathrm{n}$-dimensional overlap function.

Then the following statements hold:

1) $\mathbf{g}(x)=0$ iff $x=0$;

2) $\mathbf{g}(x)=1$ iff $x=1$.

Proof. 1) ( $\Rightarrow$ ) Since $\mathbf{h}:[0,1] \rightarrow[0,1]$ is continuous, we have that $\mathbf{h}^{n}:[0,1] \rightarrow[0,1]$ is continuous, where $\mathbf{h}^{n}$ is defined by $\mathbf{h}^{n}(x)=(\mathbf{h}(x))^{n}$ for all $x \in[0,1]$. Furthermore, we can obtain that for all $x \in[0,1]$, there exists $x^{\prime} \in[0,1]$ such that $\mathbf{h}^{n}\left(x^{\prime}\right)=x$ by items (1), (2). Thus, if $\mathbf{g}(x)=0$, then it follows that $\mathbf{g}\left(\mathbf{h}^{n}\left(x^{\prime}\right)\right)=0$ for $x=\mathbf{h}^{n}\left(x^{\prime}\right)$. Moreover, by item (3), one can get that

$$
\boldsymbol{O}_{\mathbf{g}, \mathbf{h}}\left(x^{\prime}, x^{\prime}, \cdots, x^{\prime}\right)=\mathbf{g}\left(\mathbf{h}\left(x^{\prime}\right) \mathbf{h}\left(x^{\prime}\right) \cdots \mathbf{h}\left(x^{\prime}\right)\right)=\mathbf{g}\left(\mathbf{h}^{n}\left(x^{\prime}\right)\right)=0 \text {. }
$$

Thus, using item (3) again, one has that $x^{\prime}=0$. Furthermore, using item (1) again, it follows that $x=\mathbf{h}^{n}\left(x^{\prime}\right)=\mathbf{h}^{n}(0)=0$.

$(\Leftarrow)$ If $x=0$, then, by item (3), it follows that

$$
\mathbf{g}(\mathbf{h}(x) \mathbf{h}(x) \cdots \mathbf{h}(x))=\boldsymbol{O}_{\mathbf{g}, \mathbf{h}}(x, x, \cdots, x)=0 .
$$

Furthermore, by item (1), one has that

$$
\mathbf{g}(x)=\mathbf{g}(0)=\mathbf{g}(\mathbf{h}(x) \mathbf{h}(x) \cdots \mathbf{h}(x))=0 .
$$

Hence, we have that $\mathbf{g}(x)=0$ iff $x=0$.

2) It can be proven in a similar way as item (1).

Proposition 3.4. Suppose that $F:[0,1] \rightarrow[0,1]$ is a pseudo-automorphism. Then, for any n-dimensional overlap function $\boldsymbol{O}:[0,1]^{n} \rightarrow[0,1]$, the $\mathrm{n}$-dimensional function $\boldsymbol{O}_{F}:[0,1]^{n} \rightarrow[0,1]$, given by

$$
\boldsymbol{O}_{F}\left(x_{1}, x_{2}, \cdots, x_{n}\right)=F\left(\boldsymbol{O}\left(x_{1}, x_{2}, \cdots, x_{n}\right)\right)
$$

is an n-dimensional overlap function. 
Proof. We verify that $\boldsymbol{O}_{F}$ satisfies the conditions of Definition 2.2 one by one as follows.

$\left(\boldsymbol{O}_{1}\right)$ The symmetry is obvious by the definition of $\boldsymbol{O}_{F}$

$\left(\boldsymbol{O}_{2}\right) \boldsymbol{O}_{F}\left(x_{1}, x_{2}, \cdots, x_{n}\right)=0 \Leftrightarrow F\left(\boldsymbol{O}\left(x_{1}, x_{2}, \cdots, x_{n}\right)\right)=0$

$\Leftrightarrow \boldsymbol{O}\left(x_{1}, x_{2}, \cdots, x_{n}\right)=0$

$\Leftrightarrow x_{1} x_{2} \cdots x_{n}=0$.

$\left(\boldsymbol{O}_{3}\right) \boldsymbol{O}_{F}\left(x_{1}, x_{2}, \cdots, x_{n}\right)=1 \Leftrightarrow F\left(\boldsymbol{O}\left(x_{1}, x_{2}, \cdots, x_{n}\right)\right)=1$

$\Leftrightarrow \boldsymbol{O}\left(x_{1}, x_{2}, \cdots, x_{n}\right)=1$

$\Leftrightarrow \quad x_{1} x_{2} \cdots x_{n}=1$.

$\left(\boldsymbol{O}_{4}\right)$ Since $F$ is increasing, one has that $\boldsymbol{O}_{F}$ is increasing immediately.

$\left(\boldsymbol{O}_{5}\right)$ The continuity can be obtained immediately from the continuities of $F$ and $\boldsymbol{O}$.

Proposition 3.5. Suppose that $F:[0,1] \rightarrow[0,1]$ is a pseudo-automorphism. If $(\mathbf{g}, \mathbf{h})$ is a multiplicative generator pair of $\mathrm{n}$-dimensional overlap function $\boldsymbol{O}:[0,1]^{n} \rightarrow[0,1]$, then $(F \circ \mathbf{g}, \mathbf{h})$ is a multiplicative generator pair of the n-dimensional overlap function $\boldsymbol{O}_{F}$ given in Proposition 3.4.

Proof. Since $\boldsymbol{O}$ is multiplicatively generated by the pair $(\mathbf{g}, \mathbf{h})$, we have that

$$
\boldsymbol{O}\left(x_{1}, x_{2}, \cdots, x_{n}\right)=\mathbf{g}\left(\mathbf{h}\left(x_{1}\right) \mathbf{h}\left(x_{2}\right) \cdots \mathbf{h}\left(x_{n}\right)\right) .
$$

for all $x, y \in[0,1]$. Moreover, by the definition of $\boldsymbol{O}_{P}$ it follows that for all $x, y \in[0,1]$,

$$
\begin{aligned}
\boldsymbol{O}_{F}\left(x_{1}, x_{2}, \cdots, x_{n}\right) & =F\left(\boldsymbol{O}\left(x_{1}, x_{2}, \cdots, x_{n}\right)\right) \\
& =F\left(\mathbf{g}\left(\mathbf{h}\left(x_{1}\right) \mathbf{h}\left(x_{2}\right) \cdots \mathbf{h}\left(x_{n}\right)\right)\right) \\
& =(F \circ \mathbf{g})\left(\mathbf{h}\left(x_{1}\right) \mathbf{h}\left(x_{2}\right) \cdots \mathbf{h}\left(x_{n}\right)\right)
\end{aligned}
$$

Thus, since $F$ is continuous and increasing, by Definition 3.1, we conclude that $(F \circ \mathbf{g}, \mathbf{h})$ is a multiplicative generator pair of the overlap function $\boldsymbol{O}_{F}$

Proposition 3.6. Suppose that $F:[0,1] \rightarrow[0,1]$ is a pseudo-automorphism and $\boldsymbol{O}:[0,1]^{n} \rightarrow[0,1]$ is an $\mathrm{n}$-dimensional overlap function. If $(\mathbf{g}, \mathbf{h})$ is a multiplicative generator pair of $\mathrm{n}$-dimensional overlap function $\boldsymbol{O}_{F}$ given in Proposition 3.4, then $\left(F^{-1} \circ \mathbf{g}, \mathbf{h}\right)$ is a multiplicative generator pair of the n-dimensional overlap function $O$.

Proof. Since $O_{F}$ is multiplicatively generated by the pair $(\mathbf{g}, \mathbf{h})$, one has that

$$
\boldsymbol{O}_{F}\left(x_{1}, x_{2}, \cdots, x_{n}\right)=\mathbf{g}\left(\mathbf{h}\left(x_{1}\right) \mathbf{h}\left(x_{2}\right) \cdots \mathbf{h}\left(x_{n}\right)\right) .
$$

for all $x, y \in[0,1]$. Moreover, by the definition of $\boldsymbol{O}_{F}$ it follows that for all $x, y \in[0,1]$,

$$
\begin{aligned}
\boldsymbol{O}\left(x_{1}, x_{2}, \cdots, x_{n}\right) & =\left(F^{-1} \circ F\right)\left(\boldsymbol{O}\left(x_{1}, x_{2}, \cdots, x_{n}\right)\right) \\
& =F^{-1}\left(F\left(\boldsymbol{O}\left(x_{1}, x_{2}, \cdots, x_{n}\right)\right)\right) \\
& =F^{-1}\left(\boldsymbol{O}_{F}\left(x_{1}, x_{2}, \cdots, x_{n}\right)\right) \\
& =F^{-1}\left(\mathbf{g}\left(\mathbf{h}\left(x_{1}\right) \mathbf{h}\left(x_{2}\right) \cdots \mathbf{h}\left(x_{n}\right)\right)\right) \\
& =\left(F^{-1} \circ \mathbf{g}\right)\left(\mathbf{h}\left(x_{1}\right) \mathbf{h}\left(x_{2}\right) \cdots \mathbf{h}\left(x_{n}\right)\right)
\end{aligned}
$$


Thus, since $F^{-1}$ is continuous and strictly increasing, by Definition 3.1, we conclude that $\left(F^{-1} \circ \mathbf{g}, \mathbf{h}\right)$ is a multiplicative generator pair of the n-dimensional overlap function $\boldsymbol{O}$.

\section{Homogeneity and Idempotency Property on Multiplicatively Generated n-Dimensional Overlap Functions}

Proposition 4.1. Suppose that $\boldsymbol{O}:[0,1]^{n} \rightarrow[0,1]$ is an n-dimensional overlap function multiplicatively generated by the pair $(\mathbf{g}, \mathbf{h})$. If $\mathbf{h}$ is homogeneous of order $k_{1}$ and $\mathbf{g}$ is homogeneous of order $k_{2}$, then $\boldsymbol{O}$ is homogeneous of order $n k_{1} k_{2}$.

Proof. If $\mathrm{h}$ is homogeneous of order $k_{1}$ and $\mathbf{g}$ is homogeneous of order $k_{2}$, then we can obtain that

$$
\begin{aligned}
\boldsymbol{O}\left(\alpha x_{1}, \alpha x_{2}, \cdots, \alpha x_{n}\right) & =\mathbf{g}\left(\mathbf{h}\left(\alpha x_{1}\right) \mathbf{h}\left(\alpha x_{2}\right) \cdots \mathbf{h}\left(\alpha x_{n}\right)\right) \\
& =\mathbf{g}\left(\alpha^{n k_{1}} \mathbf{h}\left(x_{1}\right) \mathbf{h}\left(x_{2}\right) \cdots \mathbf{h}\left(x_{n}\right)\right) \\
& =\left(\alpha^{n k_{1}}\right)^{k_{2}} \mathbf{g}\left(\mathbf{h}\left(x_{1}\right) \mathbf{h}\left(x_{2}\right) \cdots \mathbf{h}\left(x_{n}\right)\right) \\
& =\alpha^{n k_{1} k_{2}} \mathbf{g}\left(\mathbf{h}\left(x_{1}\right) \mathbf{h}\left(x_{2}\right) \cdots \mathbf{h}\left(x_{n}\right)\right) \\
& =\alpha^{n k_{1} k_{2}} \boldsymbol{O}\left(x_{1}, x_{2}, \cdots, x_{n}\right)
\end{aligned}
$$

Hence, it follows that $\boldsymbol{O}$ is homogeneous of order $n k_{1} k_{2}$.

Proposition 4.2. Suppose $F:[0,1] \rightarrow[0,1]$ is a $k_{1}$-homogeneous pseudoautomorphism and $\boldsymbol{O}:[0,1]^{n} \rightarrow[0,1]$ is an n-dimensional overlap function. Consider the following conditions:

1) $\boldsymbol{O}$ is homogeneous of order $k_{2}$;

2) $\boldsymbol{O}_{F}$ is homogeneous of order $k_{1} k_{2}$.

Then (1) $\Rightarrow$ (2), and if $F$ is an automorphism, then (1) $\Leftrightarrow(2)$.

Proof. (1) implies (2): If $\boldsymbol{O}$ is homogeneous of order $k_{2}$, then we get that

$$
\begin{aligned}
\boldsymbol{O}_{F}\left(\alpha x_{1}, \alpha x_{2}, \cdots, \alpha x_{n}\right) & =F\left(\boldsymbol{O}\left(\alpha x_{1}, \alpha x_{2}, \cdots, \alpha x_{n}\right)\right) \\
& =F\left(\alpha^{k_{2}} \boldsymbol{O}\left(x_{1}, x_{2}, \cdots, x_{n}\right)\right) \\
& =\left(\alpha^{k_{2}}\right)^{k_{1}} F\left(\boldsymbol{O}\left(x_{1}, x_{2}, \cdots, x_{n}\right)\right) \\
& =\alpha^{k_{1} k_{2}} \boldsymbol{O}_{F}\left(x_{1}, x_{2}, \cdots, x_{n}\right)
\end{aligned}
$$

Hence, it follows that $\boldsymbol{O}_{F}$ is homogeneous of order $k_{1} k_{2}$.

Moreover, if $F$ is an automorphism and $\boldsymbol{O}_{F}$ is homogeneous of order $k_{1} k_{2}$, then we prove item (1) as follows.

$$
\begin{aligned}
F\left(\alpha^{k_{2}} \boldsymbol{O}\left(x_{1}, x_{2}, \cdots, x_{n}\right)\right) & =\alpha^{k_{1} k_{2}} F\left(\boldsymbol{O}\left(x_{1}, x_{2}, \cdots, x_{n}\right)\right) \\
& =\alpha^{k_{1} k_{2}} \boldsymbol{O}_{F}\left(x_{1}, x_{2}, \cdots, x_{n}\right) \\
& =\boldsymbol{O}_{F}\left(\alpha x_{1}, \alpha x_{2}, \cdots, \alpha x_{n}\right) \\
& =F\left(\boldsymbol{O}\left(\alpha x_{1}, \alpha x_{2}, \cdots, \alpha x_{n}\right)\right)
\end{aligned}
$$

Hence, it follows that $\boldsymbol{O}\left(\alpha x_{1}, \alpha x_{2}, \cdots, \alpha x_{n}\right)=\alpha^{k_{2}} \boldsymbol{O}\left(x_{1}, x_{2}, \cdots, x_{n}\right)$, since $F$ is 
strictly increasing. Furthermore, we conclude that $\boldsymbol{O}$ is homogeneous of order $k_{2}$.

An element $x \in[0,1]$ is said to be an idempotent element of an $\mathrm{n}$-dimensional function $A:[0,1]^{n} \rightarrow[0,1]$ if and only if $A(x, x, \cdots, x)=x$.

Proposition 4.3. Suppose that $\boldsymbol{O}:[0,1]^{n} \rightarrow[0,1]$ is an n-dimensional overlap function multiplicatively generated by the pair $(\mathbf{g}, \mathbf{h})$, where $\mathbf{g}:[0,1] \rightarrow[0,1]$ is given by $\mathbf{g}(x)=\sqrt[n]{x}$ for all $x \in[0,1]$. Consider the following conditions:

1) $\mathbf{h}\left(x_{0}\right)=x_{0}$ for some $x_{0} \in[0,1]$;

2) $x_{0}$ is an idempotent element of $\boldsymbol{O}$.

Then $(1) \Leftrightarrow(2)$.

Proof. 1) $\Rightarrow(2)$ : If $x_{0}$ is a fixed point of $h$, then we can obtain

$$
\begin{aligned}
\boldsymbol{O}\left(x_{0}, x_{0}, \cdots, x_{0}\right) & =\mathbf{g}\left(\mathbf{h}\left(x_{0}\right) \mathbf{h}\left(x_{0}\right) \cdots \mathbf{h}\left(x_{0}\right)\right) \\
& =\sqrt[n]{\mathbf{h}\left(x_{0}\right) \mathbf{h}\left(x_{0}\right) \cdots \mathbf{h}\left(x_{0}\right)} \\
& =\mathbf{h}\left(x_{0}\right) \\
& =x_{0}
\end{aligned}
$$

Hence, it follows that $x_{0}$ is an idempotent element of $\boldsymbol{O}$.

$2) \Rightarrow$ (1): If $x_{0}$ is an idempotent element of $\boldsymbol{O}$, then one can have

$$
\begin{aligned}
x_{0} & =\boldsymbol{O}\left(x_{0}, x_{0}, \cdots, x_{0}\right) \\
& =\mathbf{g}\left(\mathbf{h}\left(x_{0}\right) \mathbf{h}\left(x_{0}\right) \cdots \mathbf{h}\left(x_{0}\right)\right) \\
& =\sqrt[n]{\mathbf{h}\left(x_{0}\right) \mathbf{h}\left(x_{0}\right) \cdots \mathbf{h}\left(x_{0}\right)} \\
& =\mathbf{h}\left(x_{0}\right)
\end{aligned}
$$

Hence, it follows that $x_{0}$ is a fixed point of $\mathbf{h}$.

Corollary 4.1. Suppose that $\boldsymbol{O}:[0,1]^{n} \rightarrow[0,1]$ is an n-dimensional overlap function multiplicatively generated by the pair $(\mathbf{g}, \mathbf{h})$, where $\mathbf{h}:[0,1] \rightarrow[0,1]$ is given by $\mathbf{h}(x)=\sqrt[n]{x}$ for all $x \in[0,1]$. Consider the following conditions:

1) $\mathbf{g}\left(x_{0}\right)=x_{0}$ for some $x_{0} \in[0,1]$;

2) $x_{0}$ is an idempotent element of $\boldsymbol{O}$.

Then $(1) \Leftrightarrow(2)$.

Proof. It can be proven in a similar way as Proposition 4.3.

Proposition 4.4. Suppose that $\boldsymbol{O}:[0,1]^{2} \rightarrow[0,1]$ is an n-dimensional overlap function and $F:[0,1] \rightarrow[0,1]$ is a pseudo-automorphism with $F\left(x_{0}\right)=x_{0}$ for some $x_{0} \in[0,1]$. Consider the following conditions:

1) $x_{0}$ is an idempotent element of $\boldsymbol{O}$;

2) $x_{0}$ is an idempotent element of $\boldsymbol{O}_{F}$;

Then (1) $\Rightarrow(2)$, and if $F$ is an automorphism, then (1) $\Leftrightarrow$ (2).

Proof. (1) $\Rightarrow$ (2): If $x_{0}$ is an idempotent element of $\boldsymbol{O}$, then we can obtain

$$
\begin{aligned}
\boldsymbol{O}_{F}\left(x_{0}, x_{0}, \cdots, x_{0}\right) & =F\left(\boldsymbol{O}\left(x_{0}, x_{0}, \cdots, x_{0}\right)\right) \\
& =F\left(x_{0}\right) \\
& =x_{0}
\end{aligned}
$$

Hence, it holds that $x_{0}$ is an idempotent element of $\boldsymbol{O}_{F}$. 
Moreover, if $F$ is an automorphism and $x_{0}$ is an idempotent element of $\boldsymbol{O}_{F}$, then we prove item (1) as follows.

$$
\begin{aligned}
F\left(x_{0}\right) & =x_{0} \\
& =\boldsymbol{O}_{F}\left(x_{0}, x_{0}, \cdots, x_{0}\right) \\
& =F\left(\boldsymbol{O}\left(x_{0}, x_{0}, \cdots, x_{0}\right)\right)
\end{aligned}
$$

Hence, we get that $x_{0}=\boldsymbol{O}\left(x_{0}, x_{0}, \cdots, x_{0}\right)$, sine $F$ is strictly increasing. Moreover, we conclude that $x_{0}$ is an idempotent element of $\boldsymbol{O}$.

\section{Conclusion}

In this paper, we mainly extend the notions of multiplicative generator pairs of overlap functions to $\mathrm{n}$-dimensional case. We propose some basic properties on multiplicatively generated $\mathrm{n}$-dimensional overlap functions, such as the homogeneity and idempotency property. In a similar way, one can also study the multiplicative generator pairs of $n$-dimensional grouping functions by the duality of n-dimensional overlap and grouping functions.

\section{Acknowledgements}

This research was supported by National Nature Science Foundation of China (Grant Nos. 61763008, 11661028, 11661030), Nature Science Foundation of Guangxi, China (Grant Nos. 2016 GXNSFAA380059, 2016 GXNSFBA380077, 2017 GXNSFAA198223), Colleges Science Research Project of Guangxi, China (Grant No. 2017 KY0264) and Scientific Research Start-up Foundation of Guilin University of Technology, China (Grant No. 002401003452).

\section{Conflicts of Interest}

The author declares no conflicts of interest regarding the publication of this paper.

\section{References}

[1] Bustince, H., Fernandez, J., Mesiar, R., Montero, J. and Orduna, R. (2009) Overlap Index, Overlap Functions and Migrativity. Proceedings of IFSA/EUSFLAT Conference, Lisbon, 20-24 July 2009, 300-305.

[2] Bustince, H., Fernandez, J., Mesiar, R., Montero, J. and Orduna, R. (2010) Overlap Functions. Nonlinear Analysis. Theory, Methods \& Applications, 72, 1488-1499. https://doi.org/10.1016/j.na.2009.08.033

[3] Bustince, H., Pagola, M., Mesiar, R., Hüllermeier, E. and Herrera, F. (2012) Grouping, Overlaps, and Generalized Bientropic Functions for Fuzzy Modeling of Pairwise Comparisons. IEEE Transactions on Fuzzy Systems, 20, 405-415. https://doi.org/10.1109/TFUZZ.2011.2173581

[4] Gómez, D., Rodríguez, J.T., Montero, J., Bustince, H. and Barrenechea, E. (2016) n-Dimensional Overlap Functions. Fuzzy Sets and Systems, 287, 57-75. https://doi.org/10.1016/j.fss.2014.11.023

[5] Dimuro, G.P., Bedregal, B., Bustince, H., Asiáin, M.J. and Mesiar, R. (2016) On Ad- 
ditive Generators of Overlap Functions. Fuzzy Sets and Systems, 287, 76-96.

https://doi.org/10.1016/j.fss.2015.02.008

[6] Qiao, J. and Hu, B.Q. (2018) On Multiplicative Generators of Overlap and Grouping Functions. Fuzzy Sets and Systems, 332, 1-24.

https://doi.org/10.1016/j.fss.2016.11.010

[7] Bustince, H., Burillo, P. and Soria, F. (2003) Automorphism, Negations and Implication Operators. Fuzzy Sets and Systems, 134, 209-229.

https://doi.org/10.1016/S0165-0114(02)00214-2 